\title{
The highly invasive Chinese mitten crab Eriocheir sinensis in the Tagus Estuary, Portugal: morphology of the specimens 20 years after the first captures
}

\author{
Dagmara Wójcik $^{1 *}$, Anna Wojtczak ${ }^{1}$, Pedro Anastácio ${ }^{2}$ and Monika Normant ${ }^{1}$ \\ ${ }^{1}$ Department of Experimental Ecology of Marine Organisms, Institute of Oceanography, University of Gdańsk, Al. Marszałka \\ J. Piłsudskiego 46, 81-378 Gdynia, Poland \\ 2 Department of Landscape, Environment and Planning, IMAR - Institute of Marine Research, University of Évora, Rua Romão \\ Ramalho 59, 7000-671 Evora, Portugal
}

Received 10 January 2014; Accepted 20 May 2014

\begin{abstract}
Eriocheir sinensis is an invasive species that spread in European waters as the result of human activity. The captures from the Portuguese population took place in September 2013 in the Tagus Estuary and were dominated by the medium-sized individuals at the beginning of their reproducing cycle $(n=192)$.
\end{abstract}

Key words: Non-native species / Crustacea / Decapoda / brackish waters

\section{Introduction}

Being a highly invasive species (Lowe et al., 2000) that may pose significant threats to freshwater and also marine ecosystems and the human economy, Eriocheir sinensis (Milne-Edwards, 18454) requires special attention. The assessment and management of non-indigenous species in new ecosystems require information on their abundance and state characterization as well as on environmental impact (Ojaveer et al., 2014). In the River Tagus, E. sinensis was noticed for the first time by local fishermen in the late 1980s (Cabral and Costa, 1999). Based on this record, it might be assumed that the time of residence of this species in Portuguese waters may be more than 30 years, making it a well-established population. Based on the invasion events of this species elsewhere in the world (e.g., Herborg et al., 2005; Veilleux and Lafontaine, 2007; Garcia-de-Lomas et al., 2010), there might be a potential risk of expanding E. sinensis invasion to other Portuguese rivers and reservoirs and also to surrounding countries. The aim of this study was to confirm the presence of a population of E. sinensis in the Tagus Estuary and to characterize its morphology, 20 years after the first register of this species in this aquatic system.

*Corresponding author: d.wojcik@ug.edu.pl

\section{Materials and methods}

Specimens of E. sinensis were caught using fyke nets (mesh: $\varnothing=20 \mathrm{~mm}$ ) in September 2013 in the upper Tagus Estuary (near Vila Franca de Xira), Portugal $(S=0$, low tide). Collections were made for the first time after 1999 (Cabral and Costa, 1999). Sampling in the upper limits of the estuary allowed us to catch the adults migrating downstream. After collection, animals $(n=192)$ were separately frozen $\left(-20^{\circ} \mathrm{C}\right)$ in string bags. After defrosting, crabs were sexed on the basis of their abdominal structures (Schäferna, 1935). The carapace width of each individual was measured using a digital calliper $( \pm 0.01 \mathrm{~mm})$, and then the wet weight of specimens was determined $( \pm 0.01 \mathrm{~g})$. Additionally, the female gonads were extracted and examined under the stereomicroscope with regard to five-scale gonad maturity stages described by Garciade-Lomas et al. (2010). To compare the size structure of male and female specimens, crabs were divided into 11 classes (every $5 \mathrm{~mm}$ width) starting at $10 \mathrm{~mm}$ (Normant et al., 2000).

The carapace length and wet weight were expressed as mean with standard deviation (mean $\pm \mathrm{SD}$ ). The relationship between carapace width and specimen wet weight was calculated according to the power equation $\left(y=a \times x^{b}\right)$. Differences in the proportion of caught males and females were tested using $\chi^{2}$, and differences between sexes in the population structure in terms of carapace width and wet weight were tested using the Kolmogorov-Smirnov test. All the analyses were conducted with SPSS 20. 
Table 1. Summary of morphological measurements of male and female Eriocheir sinensis $(\mathrm{n}=192)$ collected from the Tagus Estuary in September 2013. Data are presented as mean with standard deviation (mean $\pm \mathrm{SD}$ ). $\mathrm{F}$ - females, $\mathrm{M}$ - males, $\mathrm{n}$ - number of individuals.

\begin{tabular}{lrcc}
\hline Sex & \multicolumn{1}{c}{$n$} & Carapace width $(\mathrm{mm})$ & Wet weight $(\mathrm{g})$ \\
\hline $\mathrm{F}$ & 101 & $44.34 \pm 6.17$ & $40.08 \pm 17.92$ \\
$\mathrm{M}$ & 91 & $45.35 \pm 6.95$ & $41.11 \pm 18.44$ \\
All & $\mathbf{1 9 2}$ & $\mathbf{4 4 . 5 8} \pm \mathbf{6 . 9 2}$ & $\mathbf{4 0 . 5 7} \pm \mathbf{1 8 . 1 3}$ \\
\hline
\end{tabular}

Some crabs were damaged, sometimes missing body parts; therefore, the number of specimens used in a particular analysis (e.g., carapace width-wet weight, $n=144$ ) was lower than the total number of collected specimens.

\section{Results}

The number of individuals as well as the carapace width and wet weight of the analysed groups are given in Table 1. The difference between the proportion of females and males was not significant $\left(\chi^{2}=0.633\right.$, d.f. $=1$, $P=0.426, \chi^{2}$ test $)$. The majority of the females $(n=94)$ had gonads in the first gonad maturity stage, whereas only seven females were immature, without developed gonads.

There were no significant differences in the male and female population structures in terms of carapace width $(Z=0.533, P=0.939$, Kolmogorov-Smirnov test). The majority of the analysed crabs $(n=166)$ had a carapace width in the range $35.01-55 \mathrm{~mm}$, and the most frequent width class was $40.01-45 \mathrm{~mm}$, represented by 70 specimens (Fig. 1). There were no specimens in $15.01-20 \mathrm{~mm}$ width class. The smallest and the largest specimens were males. There were also no significant differences in the male and female population structures in terms of weight ( $Z=0.452, P=0.987$, Kolmogorov-Smirnov test).

There was a significant correlation $\left(r^{2}=0.984\right.$; $P<0.05 ; n=144$ ) between wet weight (WW) and carapace width $(\mathrm{CW})$ according to the function $\mathrm{WW}=$ $0.00047 \times \mathrm{CW}^{3.04}$.

\section{Discussion}

E. sinensis is listed among the world's worst 100 alien species (Lowe et al., 2000) and is currently viewed as an aquatic pest. The individuals from the Portuguese population captured in September 2013 were at the beginning of their reproducing cycle, dominated by the medium-sized individuals. Mean carapace width of the collected specimens was smaller than of the specimens caught in Tagus Estuary 20 years earlier (Cabral and Costa, 1999) and also than in, e.g., Polish and Spanish waters (Normant et al., 2002; Czerniejewski and Wawrzyniak, 2006; Garcia-deLomas et al., 2010) and similar to individuals from German waters (Herborg et al., 2003). The carapace width of the largest analysed specimen caught in the Tagus

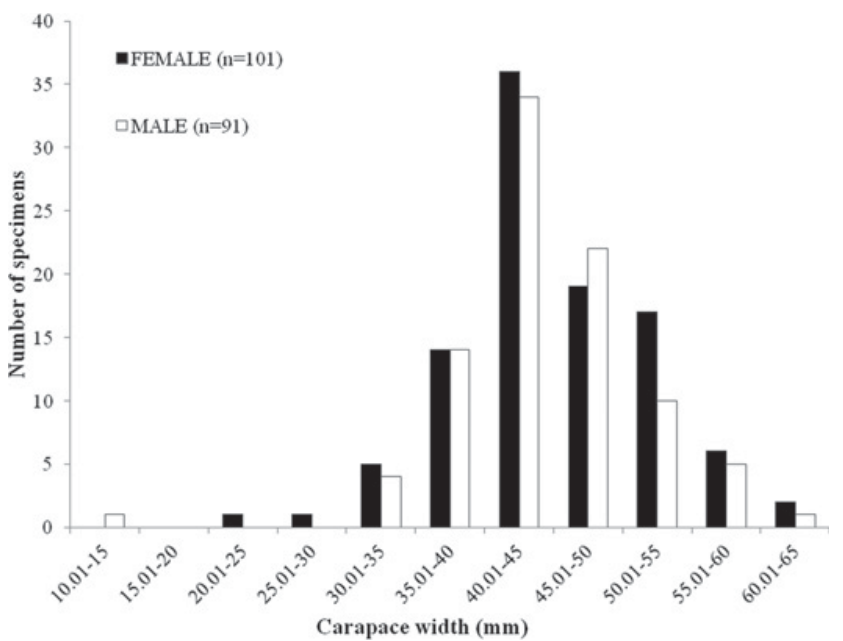

Fig. 1. Abundance of male and female Eriocheir sinensis in consecutive carapace width classes $(n=192)$ collected from the upper Tagus Estuary in September 2013.

Estuary was $63.10 \mathrm{~mm}$, the similar maximum sizes were noticed in Szczecin Lagoon, Gulf of Gdańsk, Elbe Estuary and Guadalquivir Estuary (Normant et al., 2002; Herborg et al., 2003; Czerniejewski and Wawrzyniak, 2006; Garciade-Lomas et al., 2010). This value was considerably lower than for the first captures (Cabral and Costa, 1999). What was interesting and unusual for this species, was that the analysed males were not significantly smaller than females. First observations of E. sinensis in Tagus Estuary were only adult individuals, which were caught occasionally, actually in these waters were observed established population, which consist individuals in variable sizes.

The allometric coefficient $b$ in the relationship between carapace width and wet weight was higher than for individuals from Polish waters (Normant et al., 2000; Czerniejewski and Wawrzyniak, 2006). Differences between Tagus Estuary and Southern Baltic Sea might be a result of the occurrence of an established population in Portugal, whereas in Polish waters only adult specimens are found, at the end of their life cycle, what cause significant decrease in their condition.

The percentage of males collected from the Tagus Estuary was similar to females, which is rather unusual. In most populations, males are caught more often than females (Wójcik, unpublished data). However, this tendency was also observed for the first captures (Cabral and Costa, 1999). Despite the fact that males have much greater mobility (Kobayashi and Matsuura, 1994), it is important to emphasize that the fyke-nets were in the upper limits of the estuary, and large amounts of females in this sample indicate that the reproductive migration has been started (Kobayashi and Matsuura, 1995). A peak of captures, in native and invaded waters, has been related to reproduction, e.g., increased activity related to migrations (Garcia-de-Lomas et al., 2010). None of the caught females contained eggs and their gonads were in the first developmental stadium. However, ovigerous females were previously observed in this estuary (P. Anastácio, 
unpublished data); therefore, it is clear that E. sinensis is successfully reproducing in the Tagus Estuary. Nonetheless, in the Tagus Estuary (low latitudes, warm climate) the life cycle of Chinese mitten crabs may be delayed compared to e.g., the "German" population, which reproduces in September, because of higher latitudes, where temperatures reach much lower values.

Based on Rudnick et al. (2000), individuals capable of reproduction achieve a carapace width of $35 \mathrm{~mm}$. In the analysed samples, only nine individuals (four males and five females) were not adults. Smaller specimens $(<14.13 \mathrm{~mm}$ wide) were also caught, but not analysed. The occurrence of such small individuals confirms that the population from the Tagus Estuary is established. However, there is still need for monitoring the abundance of this species, with special consideration of larvae in planctonic samples, because so far there are no data about the occurrence of any zoea or megalopa stage in Tagus waters.

It is very important to continue this research to be able to fully characterize the population of Chinese mitten crab in the Tagus Estuary. Considering the lack of knowledge about the management of E. sinensis in these waters, monitoring of this species is needed; it will help in assessing the risk of occurrence of this species in Portuguese waters, and may also help in finding solutions for preventing against $E$. sinensis invasion in other Portuguese rivers.

Acknowledgements. This research was supported by grant (no. 538-G220-B286-13) from the Faculty of Oceanography and Geography, University of Gdańsk, Poland. The research visit of Ms. D. Wójcik and A. Wojtczak at the Institute of Marine Research, Department of Landscape, Environment and Planning, University of Évora, Portugal was partially funded by the Department of Experimental Ecology of Marine Organisms (grant no. 530-G220-D427-12) and from the Faculty of Oceanography and Geography, University of Gdańsk, Poland (grant numbers 530-G000-S901-13 and 530-G000-D443-13). Also this study was partially financed by FEDER funds through the "Programa Operacional de Factores de Competitividade COMPETE" and by national funds through "FCT - Fundação para a Ciência e Tecnologia" on the scope of the Project DID (Dispersal of Invasive Decapoda; PTDC/BIABEC/105182/2008).

\section{References}

Cabral H. and Costa M., 1999. On the occurrence of the Chinese mitten crab, Eriocheir sinensis, in Portugal (Decapoda, Brachyura). Crustaceana, 72, 55-58.

Czerniejewski P. and Wawrzyniak W., 2006. Body weight, condition, and carapace width and length in the Chinese mitten crab (Eriocheir sinensis H. Milne-Edwards, 1853) collected from Szczecin Lagoon (NW Poland) in spring and autumn 2001. Oceanologia, 48, 275-285.

Garcia-de-Lomas J., Dana E., Lopez-Santiago J., Gonzalez R., Ceballos G. and Ortega F., 2010. Management of the Chinese mitten crab, Eriocheir sinensis (H. Milne-Edwards, 1853 ) in the Guadalquivir Estuary (Southern Spain). Aq. Inv., 5, 323-330.

Herborg L., Rushton S., Clare A. and Bentley M., 2003. Spread of the Chinese mitten crab (Eriocheir sinensis, H. Milne Edwards) in Continental Europe: analysis of a historical data set. Hydrobiologia, 503, 21-28.

Herborg L., Rushton S., Clare A. and Bentley M., 2005. The invasion of the Chinese mitten crab (Eriocheir sinensis) in the United Kingdom and its comparison to continental Europe. Biol. Inv., 7, 959-968.

Kobayashi S. and Matsuura S., 1994. Occurrence pattern and behavior of the Japanese mitten crab Eriocheir japanicus De Haan in the marine environment. Benthos Res., 46, 49-58.

Kobayashi S. and Matsuura S., 1995. Population structure of the Japanese mitten crab Eriocheir japanicus (De Haan) clinal variations in size and maturity. Crustacean Res., 24, $128-136$.

Lowe S., Browne M., Boudjelas S. and De Poorter M., 2000. 100 of the World's Worst Invasive Alien Species A selection from the Global Invasive Species Database. Published by The Invasive Species Specialist Group (ISSG) a specialist group of the Species Survival Commission (SSC) of the World Conservation Union, $12 \mathrm{pp}$. First published a special lift-out in Aliens 12, December 2000, Updated and reprinted version: November 2004.

Normant M., Wiszniewska A. and Szaniawska A., 2000. The Chinese mitten crab Eriocheir sinensis (Decapoda: Grapsidae) from Polish waters. Oceanologia, 42, 375-383.

Normant M., Chrobak M. and Skóra K., 2002. The Chinese mitten crab Eriocheir sinensis - an immigrant from Asia in the Gulf of Gdańsk. Oceanologia, 44, 123-125.

Ojaveer H., Galil B., Minchin D., Olenin S., Amorim A., Canning-Clode J., Chainho P., Copp G., Gollasch S., Jelmert A., Lehtiniemi M., McKenzie C., Mikus J., Miossec L., Occhipinti-Ambrogi A., Pećarević M., Pederson J., Quilez-Badia G., Wijsman J. and Zenetos A., 2014. Ten recommendations for advancing the assessment and management of non-indigenous species in marine ecosystems. Mar. Pol., 44, 160-165.

Rudnick D., Halat K. and Resh V., 2000. Distribution, ecology and potential impacts of the Chinese mitten crab (Eriocheir sinensis) in San Francisco Bay. Contrib. Water Resour. Center (University of California, Berkeley), 206, $74 \mathrm{p}$.

Schäferna K., 1935. Úvahy o èinském èi vlnoklepetém krabu. Rybáøský vistnik 15, 117-121.

Veilleux E. and de Lafontaine Y., 2007. Biological synopsis of the Chinese mitten crab (Eriocheir sinensis). Can. Manusc. Rep. Fish. Aquat. Sci., 2812, vi +45 p. 